\title{
Dampening effect of logistic source in a two-dimensional haptotaxis system with nonlinear zero-order interaction
}

\author{
Zhen Chen* \\ School of Information Science \& Technology, Donghua University, \\ Shanghai 200051, P.R. China
}

\begin{abstract}
This paper deals with the oncolytic virotherapy model

$$
\left\{\begin{array}{l}
u_{t}=\Delta u-\nabla \cdot(u \nabla v)-u z+\mu u(1-u) \\
v_{t}=-(u+w) v \\
w_{t}=D_{w} \Delta w-w+u z \\
z_{t}=D_{z} \Delta z-z-u z+\beta w
\end{array}\right.
$$

in a bounded domain $\Omega \subset \mathbb{R}^{2}$ with smooth boundary, where $\mu, D_{w}, D_{z}$ and $\beta$ are prescribed positive parameters.

For any given suitably regular initial data, the global existence of classical solution to the corresponding homogeneous Neumann initial-boundary problem for a more general model allowing $\mu=0$ was previously verified in [Y. Tao \& M. Winkler, J. Differential Equations 268 (2020), 4973-4997]. This work further shows that whenever $\mu>0$, the above-mentioned global classical solution to $(\star)$ is uniformly bounded; and moreover, if $\beta<1$, then the solution $(u, v, w, z)$ stabilizes to the constant equilibrium $(1,0,0,0)$ in the topology $L^{p}(\Omega) \times\left(L^{\infty}(\Omega)\right)^{3}$ with any $p>1$ in a large time limit.
\end{abstract}

Key words: haptotaxis; logistic source; boundedness; stabilization

MSC (2020): 35B33, 35B40, 35K57, 35Q92, 92C17

\footnotetext{
*chenzhendhu@163.com
} 


\section{Introduction}

Aim at bypassing the obstacle of drug transfer in traditional chemotherapy ([10], [6]), oncolytic virotherapy becomes an alternative treatment for cancer and it has been undergoing clinical trials (cf. [2] and [9], for instance). Very recently, in order to explore efficiency of this novel therapy, Alzahrani et al. in [1] proposed the following haptotaxis model

$$
\begin{cases}u_{t}=\Delta u-\nabla \cdot(u \nabla v)-u z+\mu u(1-u), & x \in \Omega, t>0, \\ v_{t}=-(u+w) v, & x \in \Omega, t>0, \\ w_{t}=D_{w} \Delta w-w+u z, & x \in \Omega, t>0, \\ z_{t}=D_{z} \Delta z-z-u z+\beta w, & x \in \Omega, t>0, \\ (\nabla u-u \nabla v) \cdot \nu=\frac{\partial w}{\partial \nu}=\frac{\partial z}{\partial \nu}=0, & x \in \partial \Omega, t>0, \\ u(x, 0)=u_{0}(x), \quad v(x, 0)=v_{0}(x), \quad w(x, 0)=w_{0}(x), \quad z(x, 0)=z_{0}(x), & x \in \Omega,\end{cases}
$$

with positive parameters $\mu, \beta, D_{w}$ and $D_{z}$. Throughout this work we shall assume that $\Omega \subset \mathbb{R}^{2}$ is a bounded domain with smooth boundary. Here $u, w, z$ and $v$ represent the densities of uninfected tumor cells, infected tumor cells, virus particles and normal tissue, respectively. Besides random motion, the uninfected tumor cells direct their movement toward the higher densities of tissue, they are assumed to follow a logistic growth and their number could be reduced due to infection by virus, while the infected cells randomly diffuse and have a natural death; the virus is released by infected cells and it experiences diffusion and loss due to decay and infection; and the tissue is degraded upon contact with cancer cells. The readers may refer to [1] for more detailed biological backgrounds behind (1.1).

Different from standard reaction-diffusion equations, the cross-diffusion term in chemotactic or haptotactic systems like (1.1) may exhibit a destabilizing feature ([5], 19]). Previous qualitative studies on such types of cross-diffusion systems mainly concentrate on global existence ([17], [11, [22], 21], [8]) and only a few address the large time behavior of solutions ([7, [12], [20]).

In contrast to preceding haptotaxis systems, (1.1) contains a nonlinear zero-order interaction term $u z$ in the third equation that gives rise to a new challenge in analysis of this system. Although (1.1) is indeed globally well-posed ([14]), a novel critical parameter phenomenon for infinite-time blow-up was recently detected for (1.1) without any growth or sink term ([15]). Moreover, the global existence and large time behavior of classical solutions was also discussed for (1.1) provided that $\beta<1$ and $\mu=0$ ([16]).

The purpose of this work is to identify the stabilization effect of the logistic dampening term in (1.1). To this end, we assume that

$$
\left\{\begin{array}{l}
u_{0}, v_{0} \text { and } w_{0} \text { are nonnegative functions from } C^{2+\vartheta}(\bar{\Omega}) \text { for some } \vartheta>0, \\
\text { with } u_{0}>0, w_{0} \neq \equiv, z_{0} \not \equiv 0, \sqrt{v_{0}} \in W^{1,2}(\Omega) \text { and } \frac{\partial u_{0}}{\partial \nu}=\frac{\partial v_{0}}{\partial \nu}=\frac{\partial w_{0}}{\partial \nu}=0 \text { on } \partial \Omega .
\end{array}\right.
$$

We shall prove that whenever $\mu>0$, for any $\beta>0$ the classical solution of (1.1) is uniformly bounded; furthermore, it is claimed that if $\beta<1$, then the solution $(u, v, w, z)$ stabilizes to the constant stationary solution $(1,0,0,0)$ in $L^{\infty}$ topology. More precisely, we have the following: 
Theorem 1.1 Let $\mu>0$, and let $\Omega \subset \mathbb{R}^{2}$ be a smoothly bounded domain. Then for any given $\left(u_{0}, v_{0}, w_{0}, z_{0}\right)$ satisfying (1.2), the solution $(u, v, w, z)$ of (1.1) is bounded in the sense that

$$
\sup _{t>0}\left\{\|u(\cdot, t)\|_{L^{\infty}(\Omega)}+\|v(\cdot, t)\|_{L^{\infty}(\Omega)}+\|w(\cdot, t)\|_{L^{\infty}(\Omega)}+\|z(\cdot, t)\|_{L^{\infty}(\Omega)}\right\}<\infty .
$$

If

$$
\beta \in(0,1)
$$

then furthermore

$$
u(\cdot, t) \rightarrow 1 \quad \text { in } L^{p}(\Omega) \quad \text { for all } p \geq 1
$$

and

$$
v(\cdot, t) \rightarrow 0 \quad \text { in } L^{\infty}(\Omega)
$$

as well as

$$
w(\cdot, t) \rightarrow 0 \quad \text { in } L^{\infty}(\Omega)
$$

and

$$
z(\cdot, t) \rightarrow 0 \quad \text { in } L^{\infty}(\Omega)
$$

as $t \rightarrow \infty$.

\section{$2 \quad L^{p}$ bounds for $w$}

Let us begin with the global smooth solvability of (1.1) previously asserted in [14, together with one basic solution property.

Lemma 2.1 Let $\Omega \subset \mathbb{R}^{2}$ be a bounded domain with smooth boundary, and assume that $\left(u_{0}, v_{0}, w_{0}, z_{0}\right)$ fulfills (1.2). Then the problem (1.1) admits a uniquely determined classical solution $(u, v, w, z) \in$ $\left(C^{2,1}(\bar{\Omega} \times[0, \infty))\right)^{4}$ for which $v$ is nonnegative, and for which $u, w$ and $z$ are positive in $\bar{\Omega} \times(0, \infty)$. Moreover,

$$
\|v(\cdot, t)\|_{L^{\infty}(\Omega)} \leq\left\|v_{0}(\cdot)\right\|_{L^{\infty}(\Omega)} \quad \text { for all } t>0 .
$$

The presence of logistic dampening in the first equation in (1.1) will be decisive for us to further verify the uniform boundedness of the global solution constructed in Lemma 2.1, and correspondingly the assumption that $\mu>0$ will play a key role in deriving the following $L^{1}$ bounds for $u, w$ and $z$ and a space-time $L^{2}$ bound for $u$, which serve as a starting point of our subsequently reasoning. 
Lemma 2.2 There exists $C>0$ such that

$$
\int_{\Omega} u(\cdot, t) \leq C, \quad \int_{\Omega} w(\cdot, t) \leq C \quad \text { and } \quad \int_{\Omega} z(\cdot, t) \leq C
$$

as well as

$$
\int_{t}^{t+1} \int_{\Omega} u^{2} \leq C
$$

for all $t>0$.

Proof. We firstly assume that $\beta>0$ and use (1.1) to compute

$$
\begin{aligned}
\frac{d}{d t}\left\{2 \beta \int_{\Omega} u+2 \beta \int_{\Omega} w+\int_{\Omega} z\right\}= & \left\{-2 \beta \int_{\Omega} u z+2 \beta \mu \int_{\Omega} u-2 \beta \mu \int_{\Omega} u^{2}\right\} \\
& +\left\{-2 \beta \int_{\Omega} w+2 \beta \int_{\Omega} u z\right\} \\
& +\left\{-\int_{\Omega} z-\int_{\Omega} u z+\beta \int_{\Omega} w\right\} \\
= & 2 \beta \mu \int_{\Omega} u-\beta \int_{\Omega} w-\int_{\Omega} z-\int_{\Omega} u z-2 \beta \mu \int_{\Omega} u^{2}
\end{aligned}
$$

for all $t>0$, so that by Young's inequality,

$$
\begin{aligned}
\frac{d}{d t}\left\{2 \beta \int_{\Omega} u+2 \beta \int_{\Omega} w+\int_{\Omega} z\right\} & +\frac{1}{2} \cdot\left\{2 \beta \int_{\Omega} u+2 \beta \int_{\Omega} w+\int_{\Omega} z\right\}+\beta \mu \int_{\Omega} u^{2} \\
& =-\beta(1+2 \mu) \int_{\Omega} u-\beta \mu \int_{\Omega} u^{2}-\frac{1}{2} \int_{\Omega} z-\int_{\Omega} u z \\
& \leq-\beta(1+2 \mu) \int_{\Omega} u-\beta \mu \int_{\Omega} u^{2} \\
& \leq \frac{\beta(1+2 \mu)^{2}}{4 \mu} \cdot|\Omega| \text { for all } t>0 .
\end{aligned}
$$

Upon an ODE comparison, this readily implies (2.2) due to $\beta>0$ and thereby a further integration over time yields (2.3) thanks to the fact that $\mu>0$. If $\beta=0$, by estimating $\frac{d}{d t}\left\{\int_{\Omega} w+\int_{\Omega} z\right\}$ and $\frac{d}{d t} \int_{\Omega} u$ we still can arrive at (2.2) and (2.3) in a same manner.

With the help of parabolic smooth properties in the spatial two-dimensional setting, the $L^{1}$ bound of $w$ actually implies $L^{p}$ integrability of $z$ for arbitrarily large finite $\mathrm{p}$.

Lemma 2.3 Let $p \in(1, \infty)$. Then there exists $C(p)>0$ such that

$$
\|z(\cdot, t)\|_{L^{p}(\Omega)} \leq C(p) \quad \text { for all } t>0 .
$$

Proof. By nonnegativity of $u z$ and $z$, in light of the order preserving property of $e^{\sigma \Delta}$ for $\sigma \geq 0$ we firstly find that

$$
\begin{aligned}
z(\cdot, t) & =e^{t\left(D_{z} \Delta-1\right)} z_{0}-\int_{0}^{t} e^{(t-s)\left(D_{z} \Delta-1\right)} u(\cdot, s) z(\cdot, s) d s+\beta \int_{0}^{t} e^{(t-s)\left(D_{z} \Delta-1\right)} w(\cdot, s) d s \\
& \leq e^{t D_{z} \Delta} z_{0}+\beta \int_{0}^{t} e^{(t-s)\left(D_{z} \Delta-1\right)} w(\cdot, s) d s \quad \text { in } \Omega \quad \text { for all } t>0,
\end{aligned}
$$


and hence, by well-known smoothing properties of the Neumann heat semigroup $\left(e^{\sigma \Delta}\right)_{\sigma \geq 0}([18])$ and Lemma 2.2, secondly see that

$$
\begin{aligned}
\|z(\cdot, t)\|_{L^{p}(\Omega)} & \leq\left\|e^{t D_{z} \Delta} z_{0}\right\|_{L^{p}(\Omega)}+c_{1} \beta \int_{0}^{t}\left(1+(t-s)^{-1+\frac{1}{p}}\right) e^{-(t-s)}\|w(\cdot, s)\|_{L^{1}(\Omega)} d s \\
& \leq\left\|z_{0}\right\|_{L^{p}(\Omega)}+c_{1} c_{2} \beta \int_{0}^{t}\left(1+(t-s)^{-1+\frac{1}{p}}\right) e^{-(t-s)} d s \quad \text { for all } t>0
\end{aligned}
$$

with some $c_{1}>0$ and $c_{2}>0$. This entails (2.4), because

$$
\int_{0}^{t}\left(1+(t-s)^{-1+\frac{1}{p}}\right) e^{-(t-s)} d s \leq \int_{0}^{\infty}\left(1+\sigma^{-1+\frac{1}{p}}\right) e^{-\sigma} d \sigma<2+p \quad \text { for all } t>0 .
$$

In light of the above $L^{p}$ integrability information of $z$ along with a space-time $L^{2}$ bound for $u$ in (2.3) and according to parabolic smooth estimates in the two-dimensional case once again, we also obtain $L^{p}$ bounds of $w$ for arbitrary $p>2$.

Lemma 2.4 Let $p \in(2, \infty)$. Then there exists $C(p)>0$ with the property

$$
\|w(\cdot, t)\|_{L^{p}(\Omega)} \leq C(p) \quad \text { for all } t>0 .
$$

Proof. Given $p>2$, we choose $q:=\frac{2 p}{p+1} \in(1,2)$. We then recall known smoothing estimates for the Neumann heat semigroup on $\Omega([18])$ to obtain positive constants $c_{1}=c_{1}(p)$ and $c_{2}=c_{2}(p)$ satisfying

$$
\left\|e^{D_{w} \Delta} \varphi\right\|_{L^{p}(\Omega)} \leq c_{1}\|\varphi\|_{L^{1}(\Omega)} \quad \text { for all } \varphi \in C^{0}(\bar{\Omega})
$$

and

$$
\left\|e^{\sigma D_{w} \Delta} \varphi\right\|_{L^{p}(\Omega)} \leq c_{2} \sigma^{-\frac{p-1}{2 p}}\|\varphi\|_{L^{q}(\Omega)} \quad \text { for all } \sigma \in(0,1) \text { and each } \varphi \in C^{0}(\bar{\Omega}) .
$$

Now in light of a variation-of-constants representation of $w$ solving the third equation in (1.1), we can invoke (2.6) and (2.7) to estimate

$$
\begin{aligned}
\|w(\cdot, t)\|_{L^{p}(\Omega)} & =\left\|e^{D_{w} \Delta-1} w(\cdot, t-1)+\int_{t-1}^{t} e^{(t-s)\left(D_{w} \Delta-1\right)} u(\cdot, s) z(\cdot, s) d s\right\|_{L^{p}(\Omega)} \\
& \leq e^{-1} \cdot c_{1}\|w(\cdot, t-1)\|_{L^{1}(\Omega)}+c_{2} \int_{t-1}^{t}(t-s)^{-\frac{p-1}{2 p}}\|u(\cdot, s) z(\cdot, s)\|_{L^{q}(\Omega)} d s
\end{aligned}
$$

for all $t>1$, where according to the Hölder inequality and Lemma 2.3, we find $c_{3}=c_{3}(p)>0$ such that

$$
\begin{aligned}
c_{2} \int_{t-1}^{t}(t-s)^{-\frac{p-1}{2 p}\|u(\cdot, s) z(\cdot, s)\|_{L^{q}(\Omega)} d s} & \leq c_{2} \int_{t-1}^{t}(t-s)^{-\frac{p-1}{2 p}}\|u(\cdot, s)\|_{L^{2}(\Omega)}\|z(\cdot, s)\|_{L^{\frac{2 q}{2-q}(\Omega)}} d s \\
& \leq c_{3} \int_{t-1}^{t}(t-s)^{-\frac{p-1}{2 p}}\|u(\cdot, s)\|_{L^{2}(\Omega)} d s \\
& \leq c_{3} \cdot\left\{\int_{t-1}^{t}(t-s)^{-\frac{p-1}{p}} d s\right\}^{\frac{1}{2}} \cdot\left\{\int_{t-1}^{t}\|u(\cdot, s)\|_{L^{2}(\Omega)}^{2} d s\right\}^{\frac{1}{2}} \\
& =p c_{3} \cdot\left\{\int_{t-1}^{t}\|u(\cdot, s)\|_{L^{2}(\Omega)}^{2} d s\right\}^{\frac{1}{2}} \text { for all } r>1 .
\end{aligned}
$$


Since

$$
\sup _{t>1}\|w(\cdot, t-1)\|_{L^{1}(\Omega)}<\infty \quad \text { and } \quad \int_{t-1}^{t}\|u(\cdot, s)\|_{L^{2}(\Omega)}^{2} d s=\int_{t-1}^{t} \int_{\Omega} u^{2}<\infty \quad \text { for all } t>1
$$

by Lemma 2.2, (2.8) yields (2.5), because $w$ is bounded in $\bar{\Omega} \times[0,1]$ by Lemma 2.1 .

\section{$3 \quad L^{\infty}$ estimates on $u$}

In this section we shall establish the boundedness of $u$ in $L^{\infty}$. For this purpose, as performed in [3], [4], [17] and [1] which dealt with haptotaxis-related systems, we introduce the variable change

$$
a:=u e^{-v}
$$

and then in view of (1.1),

$$
\begin{cases}a_{t}=e^{-v} \nabla \cdot\left(e^{v} \nabla a\right)+a\left(a e^{v}+w\right) v-a z+\mu a\left(1-a e^{v}\right), & x \in \Omega, t>0, \\ v_{t}=-\left(a e^{v}+w\right) v, & x \in \Omega, t>0, \\ \frac{\partial a}{\partial \nu}=0, & x \in \partial \Omega, t>0, \\ a(x, 0)=u_{0}(x) e^{-v_{0}(x)}=: a_{0}(x), \quad v(x, 0)=v_{0}(x), & x \in \Omega .\end{cases}
$$

A direct testing procedure leads to the following primary inequality which will be invoked in Lemma 3.2 and Lemma 3.4 below.

Lemma 3.1 Let $p>1$. Then

$$
\frac{d}{d t} \int_{\Omega} e^{v} a^{p} \leq-p(p-1) \int_{\Omega} e^{v} a^{p-2}|\nabla a|^{2}+(p-1) \int_{\Omega} e^{v} a^{p}\left(a e^{v}+w\right) v+\mu p \int_{\Omega} e^{v} a^{p} \quad \text { for all } t>0 .
$$

Proof. From (3.2) we infer that $\left(a e^{v}+w\right) v=-v_{t}$ in $\Omega \times(0, \infty)$ and that

$$
\begin{aligned}
\frac{d}{d t} \int_{\Omega} e^{v} a^{p}= & p \int_{\Omega} e^{v} a^{p-1} \cdot\left\{e^{-v} \nabla \cdot\left(e^{v} \nabla a\right)-a v_{t}-a z+\mu a-\mu a^{2} e^{v}\right\}+\int_{\Omega} e^{v} a^{p} v_{t} \\
= & -p(p-1) \int_{\Omega} e^{v} a^{p-2}|\nabla a|^{2}-(p-1) \int_{\Omega} e^{v} a^{p} v_{t}+\mu p \int_{\Omega} e^{v} a^{p} \\
& -\rho p \int_{\Omega} e^{v} a^{p} z-\mu p \int_{\Omega} e^{2 v} a^{p+1}
\end{aligned}
$$

for all $t>0$, this leads to (3.3) upon abandoning the rightmost two nonpositive summands.

Using a bootstrap $L^{p}$-estimate technique developed in [13, Lemma 3.8] and relying on (2.3), Lemma 2.4 and Lemma 2.1, an application of Lemma 3.1 to $p=2$ can yield a bound for $a$ in $L^{2}$.

Lemma 3.2 There exists $C>0$ fulfilling

$$
\|a(\cdot, t)\|_{L^{2}(\Omega)} \leq C \quad \text { for all } t>0 .
$$


Proof. Lemma 2.1 guarantees the existence of $c_{1}>0$ such that

$$
\|v(\cdot, t)\|_{L^{\infty}(\Omega)} \leq c_{1} \quad \text { for all } t>0
$$

whereas Lemma 2.4 provides $c_{2}>0$ satisfying

$$
\int_{\Omega} w^{3}(\cdot, t) \leq c_{2} \quad \text { for all } t>0 .
$$

Now from Lemma 3.1 we infer the inequality

$$
\frac{d}{d t} \int_{\Omega} e^{v} a^{2}+2 \int_{\Omega} e^{v}|\nabla a|^{2} \leq \int_{\Omega} e^{2 v} a^{3} v+\int_{\Omega} e^{v} a^{2} v w+2 \mu \int_{\Omega} e^{v} a^{2} \quad \text { for all } t>0
$$

where by Young's inequality, (3.5) and (3.6),

$$
\begin{aligned}
\int_{\Omega} e^{2 v} a^{3} v+\int_{\Omega} e^{v} a^{2} v w+2 \mu \int_{\Omega} e^{v} a^{2} & \leq c_{1} e^{2 c_{1}} \int_{\Omega} a^{3}+c_{1} e^{c_{1}} \int_{\Omega} a^{2} w+2 \mu e^{c_{1}} \int_{\Omega} a^{2} \\
& \leq\left\{c_{1} e^{2 c_{1}}+c_{1} e^{c_{1}}+2 \mu e^{c_{1}}\right\} \cdot \int_{\Omega} a^{3}+c_{1} e^{c_{1}} \int_{\Omega} w^{3}+2 \mu e^{c_{1}} \cdot|\Omega| \\
& \leq c_{3} \int_{\Omega} a^{3}+c_{4} \quad \text { for all } t>0
\end{aligned}
$$

with $c_{3}:=c_{1} e^{2 c_{1}}+c_{1} e^{c_{1}}+2 \mu e^{c_{1}}$ and $c_{4}:=c_{1} e^{c_{1}} c_{2}+2 \mu e^{c_{1}} \cdot|\Omega|$. As the Gagliardo-Nirenberg inequality along with Young's inequality yields that

$$
\begin{aligned}
c_{3} \int_{\Omega} a^{3}=c_{3}\|a\|_{L^{3}(\Omega)}^{3} & \leq c_{5}\|\nabla a\|_{L^{2}(\Omega)}\|a\|_{L^{2}(\Omega)}^{2}+c_{5}\|a\|_{L^{2}(\Omega)}^{3} \\
& \leq\left\{2\|\nabla a\|_{L^{2}(\Omega)}^{2}+c_{5}^{2}\|a\|_{L^{2}(\Omega)}^{4}\right\}+\left\{\|a\|_{L^{2}(\Omega)}^{4}+c_{5}^{4}\right\} \\
& \leq 2 \int_{\Omega} e^{v}|\nabla a|^{2}+c_{6}\|a\|_{L^{2}(\Omega)}^{4}+c_{6} \quad \text { for all } t>0
\end{aligned}
$$

with some $c_{5}>0$ and $c_{6}:=\max \left\{c_{5}^{2}+1, c_{5}^{4}\right\}$, by $\int_{\Omega} a^{2} \leq \int_{\Omega} e^{v} a^{2}$ we thus infer from (3.7) that

$$
\frac{d}{d t} \int_{\Omega} e^{v} a^{2} \leq\left\{c_{6} \int_{\Omega} a^{2}(\cdot, t)\right\} \cdot \int_{\Omega} e^{v} a^{2}+c_{6} \quad \text { for all } t>0
$$

Letting

$$
h(t):=c_{6} \int_{\Omega} a^{2}(\cdot, t) \quad \text { for all } t>0,
$$

We may apply Lemma 2.2 along with (2.1) to find $c_{7}>0$ fulfilling

$$
\int_{t}^{t+1} h(s) d s \leq c_{7} \quad \text { for all } t>0,
$$


and thereby for any given $t>1$, it is possible to fix $t_{0}=t_{0}(t) \in(t-1, t)$ such that $t_{0}>0$ and

$$
\int_{\Omega} a^{2}\left(\cdot, t_{0}\right) \leq c_{8}:=\max \left\{\int_{\Omega} a_{0}^{2}, c_{7}\right\}
$$

so that integrating (3.8) shows that

$$
\begin{aligned}
\int_{\Omega} e^{v(\cdot, t)} a^{2}(\cdot, t) & \leq\left(\int_{\Omega} e^{v\left(\cdot, t_{0}\right)} a^{2}\left(\cdot, t_{0}\right)\right) \cdot e^{\int_{t_{0}}^{t} h(s) d s}+c_{6} \int_{t_{0}}^{t} e^{\int_{s}^{t} h(\sigma) d \sigma} d s \\
& \leq e^{c_{1}} c_{8} \cdot e^{c_{7}}+c_{6} e^{c_{7}} \quad \text { for all } t>1
\end{aligned}
$$

due to (3.5), (3.9) and the fact that $t-t_{0} \leq 1$. Since $e^{v} \geq 1$ and $a$ is bounded in $\bar{\Omega} \times[0,1]$ by Lemma 2.1, this proves (3.4).

Using this $L^{2}$ bound of a together with the smoothing properties of the Neumann heat semigroup on $\Omega$ once more, we can further improve the regularity of $w$ and $z$ in the following sense.

Lemma 3.3 There exists $C>0$ with the property that

$$
\|w(\cdot, t)\|_{W^{1,3}(\Omega)} \leq C \quad \text { for all } t>0
$$

and

$$
\|z(\cdot, t)\|_{W^{1,3}(\Omega)} \leq C \quad \text { for all } t>0 .
$$

Proof. Combine Lemma 3.2, Lemma 2.4 and Lemma 2.3 with (3.1) and (2.1) we can fix positive constants $c_{1}, c_{2}$ and $c_{3}$ satisfying

$$
\|u(\cdot, t)\|_{L^{2}(\Omega)} \leq c_{1}, \quad\|w(\cdot, t)\|_{L^{2}(\Omega)} \leq c_{2} \quad \text { and } \quad\|z(\cdot, t)\|_{L^{4}(\Omega)} \leq c_{3} \quad \text { for all } t>0 .
$$

Since well-known smoothing properties of the Neumann heat semigroup on $\Omega$ ([18]) ensure the existence of $c_{4}>0$ and $c_{5}>0$ such that

$$
\left\|e^{D_{w} \Delta} \varphi\right\|_{W^{1,3}(\Omega)} \leq c_{4}\|\varphi\|_{L^{2}(\Omega)} \quad \text { for all } \varphi \in C^{0}(\bar{\Omega})
$$

and

$$
\left\|e^{\sigma D_{w} \Delta} \varphi\right\|_{W^{1,3}(\Omega)} \leq c_{5} \sigma^{-\frac{11}{12}}\|\varphi\|_{L^{\frac{4}{3}}(\Omega)} \quad \text { for all } \sigma \in(0,1) \text { and any } \varphi \in C^{0}(\bar{\Omega}),
$$

we thus see that by the Hölder inequality,

$$
\begin{aligned}
\|w(\cdot, t)\|_{W^{1,3}(\Omega)} & =\left\|e^{D_{w} \Delta-1} w(\cdot, t-1)+\int_{t-1}^{t} e^{(t-s)\left(D_{w} \Delta-1\right)} u(\cdot, s) z(\cdot, s) d s\right\|_{W^{1,3}(\Omega)} \\
& \leq c_{4} e^{-1}\|w(\cdot, t-1)\|_{L^{2}(\Omega)}+c_{5} \int_{t-1}^{t}(t-s)^{-\frac{11}{12}}\|u(\cdot, s) z(\cdot, s)\|_{L^{\frac{4}{3}}(\Omega)} d s \\
& \leq c_{4} e^{-1}\|w(\cdot, t-1)\|_{L^{2}(\Omega)}+c_{5} \int_{t-1}^{t}(t-s)^{-\frac{11}{12}}\|u(\cdot, s)\|_{L^{2}(\Omega)}\|z(\cdot, s)\|_{L^{4}(\Omega)} d s \\
& \leq c_{2} c_{4} e^{-1}+12 c_{1} c_{3} c_{5} \quad \text { for all } t>1,
\end{aligned}
$$


which entails (3.10), while (3.11) can be proved in a quite similar manner.

Lemma 3.3 actually implies the boundedness of $w$ in $L^{\infty}$ due to the Sobolev embedding $W^{1, p}(\Omega) \hookrightarrow$ $C^{0}(\bar{\Omega})$ for $p>n$, with $n$ denoting the space dimension. Depending on this and Lemma 3.2, we can achieve the boundedness of $a$ in $L^{\infty}$ via a Moser iteration technique.

Lemma 3.4 There exists $C>0$ such that

$$
\|a(\cdot, t)\|_{L^{\infty}(\Omega)} \leq C \quad \text { for all } t>0 .
$$

Proof. According to Lemma 2.1 and Lemma 3.3 along with the Sobolev embedding $W^{1,3}(\Omega) \hookrightarrow$ $C^{0}(\bar{\Omega})$, we can pick $c_{1}>0$ and $c_{2}>0$ such that $v \leq c_{1}$ and $w \leq c_{2}$ on $\Omega \times(0, \infty)$. From Lemma 3.1 along with Young's inequality we next can infer that for all $p>1$,

$$
\frac{d}{d t} \int_{\Omega} e^{v} a^{p}+3 \int_{\Omega}\left|\nabla a^{\frac{p}{2}}\right|^{2}+\int_{\Omega} e^{v} a^{p} \leq c_{3} p \int_{\Omega} a^{p+1}+c_{4} \quad \text { for all } t>0
$$

with $c_{3}>0$ and $c_{4}>0$ which are independent of $p$. Since Lemma 3.2 provides $c_{5}>0$ such that

$$
\int_{\Omega} a^{2}(\cdot, t) \leq c_{5} \quad \text { for all } t>0
$$

we thus can invoke a Moser-type iteration method to derive (3.12) (cf. [16, Lemma 6.4] for details).

\section{Stabilization when $\beta<1$}

Throughout this section we shall suppose that $\beta<1$, and we shall investigate the asymptotic behavior of the global classical solution constructed in Lemma 2.1. In fact, the assumption that $\beta<1$ readily results in the exponential decay property of $w$ and $z$.

Lemma 4.1 Let $\beta<1$. Then there exist $\gamma \in(0,1)$ and $C>0$ such that

$$
\|w(\cdot, t)\|_{L^{\infty}(\Omega)} \leq C e^{-\gamma t} \quad \text { for all } t>0
$$

and

$$
\|z(\cdot, t)\|_{L^{\infty}(\Omega)} \leq C e^{-\gamma t} \quad \text { for all } t>0
$$

Proof. We use the third and fourth equations in (1.1) to compute

$$
\begin{aligned}
\frac{d}{d t}\left\{\int_{\Omega} w+\int_{\Omega} z\right\} & =\left\{-\int_{\Omega} w+\int_{\Omega} u z\right\}+\left\{-\int_{\Omega} z-\int_{\Omega} u z+\beta \int_{\Omega} w\right\} \\
& =-(1-\beta) \int_{\Omega} w-\int_{\Omega} z \\
& \leq-(1-\beta) \cdot\left\{\int_{\Omega} w+\int_{\Omega} z\right\} \quad \text { for all } t>0
\end{aligned}
$$


which implies that

$$
\int_{\Omega} w+\int_{\Omega} z \leq\left\{\int_{\Omega} w_{0}+\int_{\Omega} z_{0}\right\} \cdot e^{-(1-\beta) t} \quad \text { for all } t>0 .
$$

This in conjunction with Lemma the Gagliardo-Nirenberg inequality and Lemma 3.3 yields $c_{1}>0$ and $c_{2}>0$ such that

$$
\begin{aligned}
\|w(\cdot, t)\|_{L^{\infty}(\Omega)} & \leq c_{1}\|w(\cdot, t)\|_{W^{1,3}(\Omega)}^{\frac{6}{7}}\|w(\cdot, t)\|_{L^{1}(\Omega)}^{\frac{1}{7}} \\
& \leq c_{2} e^{-\frac{1-\beta}{7} t} \quad \text { for all } t>0,
\end{aligned}
$$

and thus the proof is complete with $\gamma:=\frac{1-\beta}{7}$ and $C:=c_{2}$.

Since $z$ eventually vanishes in $L^{\infty}$ due to (4.2), from (3.2) and the parabolic comparison principle we can infer that $a$ has a positive lower bound, and thus so does $u$ in view of (3.1) and (2.1).

Lemma 4.2 If $\beta<1$, then one can find $\delta>0$ fulfilling

$$
u(x, t) \geq \delta \quad \text { for all }(x, t) \in \Omega \times(0, \infty) .
$$

Proof. According to Lemma 4.1 and Lemma 2.1, it is possible for us to pick $t_{0}>0$ and $c_{1}>0$ such that

$$
z(x, t) \leq c_{1} e^{-\gamma t} \leq \frac{\mu}{2} \quad \text { for all } x \in \Omega \text { and } t>t_{0} .
$$

From the first equation in (3.2) along with this and Lemma 2.1 once again we infer that $a$ satisfies

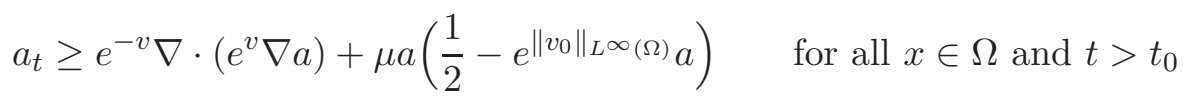

with $\frac{\partial a}{\partial \nu}=0$ throughout $\partial \Omega \times\left(t_{0}, \infty\right)$. On the other hand, for

$$
\underline{a}(x, t):=y(t), \quad x \in \bar{\Omega}, t \geq t_{0},
$$

with $y \in C^{1}\left(\left[t_{0}, \infty\right)\right)$ denoting the solution of

$$
\left\{\begin{array}{l}
y^{\prime}(t)=\mu y\left(\frac{1}{2}-c_{2} y\right), \quad t>t_{0} \\
y\left(t_{0}\right):=\inf _{x \in \Omega} a\left(x, t_{0}\right)>0
\end{array}\right.
$$

where $c_{2}:=e^{\left\|v_{0}\right\|_{L^{\infty}(\Omega)}}$. Then we have

$$
\begin{aligned}
\underline{a}_{t}-e^{-v(x, t)} \nabla \cdot\left(e^{v(x, t)} \nabla \underline{a}\right)-\mu \underline{a}\left(\frac{1}{2}-c_{2} \underline{a}\right) \\
=y^{\prime}(t)-\mu y\left(\frac{1}{2}-c_{2} y\right) \\
=0 \quad \text { for all } x \in \Omega \text { and } t>t_{0},
\end{aligned}
$$


and since clearly $\frac{\partial a}{\partial \nu}=0$ on $\partial \Omega \times\left(t_{0}, \infty\right)$ and

$$
\underline{a}\left(x, t_{0}\right)=y\left(t_{0}\right)=\inf _{x \in \Omega} a\left(x, t_{0}\right) \leq a\left(x, t_{0}\right) \quad \text { for all } x \in \Omega,
$$

an application of the comparison principle readily shows that $a \geq \underline{a}$ in $\Omega \times\left(t_{0}, \infty\right)$ and hence

$$
u(x, t)=a(x, t) e^{v(x, t)} \geq a(x, t) \geq y(t) \quad \text { for all } x \in \Omega \text { and } t>t_{0} .
$$

Since we have $y(t) \rightarrow \frac{1}{2 c_{2}}$ as $t \rightarrow \infty$ due to $y\left(t_{0}\right)>0$, we can conclude that there exists $t_{1} \geq t_{0}$ such that

$$
y(t) \rightarrow \frac{1}{4 c_{2}} \quad \text { for all } t>t_{1} .
$$

Finally, the positivity and continuity of $u(x, t)$ in $\Omega \times(0, \infty)$ by Lemma 2.1 guarantees the existence of $c_{3}>0$ fulfilling

$$
c_{3}:=\min _{\bar{\Omega} \times\left[0, t_{1}\right]} u(x, t)>0 .
$$

So that (4.3) results from (4.4)-(4.6) with $\delta:=\min \left\{\frac{1}{4 c_{2}}, c_{3}\right\}>0$.

According to the second equation in (1.1), Lemma 4.2 along with Lemma 2.1 immediately leads to the following.

Corollary 4.3 Let $\beta<1$. Then we have

$$
v(x, t) \leq\left\|v_{0}(\cdot)\right\|_{L^{\infty}(\Omega)} \cdot e^{-\delta t} \quad \text { for all } x \in \Omega \text { and } t>0,
$$

where $\delta>0$ is defined by Lemma 4.2.

Proof. $\quad$ From (1.1), Lemma 4.2 and the nonnegativity of $v$ and $w$ we obtain that

$$
v_{t}=-(u+w) v \leq-\delta v \quad \text { for all } x \in \Omega \text { and } t>0,
$$

from which (4.7) readily follows.

With the outcomes of Lemma 3.4. Lemma 4.1 and Corollary 4.3, we firstly obtain the following weak convergence information of $a$, which is based on a specific testing procedure.

Lemma 4.4 If $\beta<1$, then there holds

$$
\int_{0}^{\infty} \int_{\Omega}\left(a e^{v}-1\right)^{2}<\infty
$$

Proof. Clearly, Lemma 3.4, Lemma 4.1 and Corollary 4.3 ensure the existence of $c_{1}>0, c_{2}>0$, $c_{3}>0, c_{4}>0$ and $c_{5}>0$ such that

$$
a \leq c_{1}, \quad v \leq c_{2}, \quad \text { and } \quad w \leq c_{3} \quad \text { in } \Omega \times(0, \infty)
$$

as well as

$$
\int_{0}^{\infty} \int_{\Omega} v \leq c_{4} \text { and } \int_{0}^{\infty} \int_{\Omega} z \leq c_{5} .
$$


According to the basic inequality: $s-1-\ln s \geq 0$ for all $s>0$ and in view of the fact that $v_{t} \leq 0$, we use (3.2) to estimate

$$
\begin{aligned}
\frac{d}{d t} \int_{\Omega} e^{v}(a-1-\ln a)= & \int_{\Omega} e^{v}\left(1-\frac{1}{a}\right) a_{t}+\int_{\Omega} e^{v}(a-1-\ln a) v_{t} \\
\leq & \int_{\Omega} e^{v}\left(1-\frac{1}{a}\right) a_{t} \\
= & \int_{\Omega} e^{v}\left(1-\frac{1}{a}\right) \cdot\left\{e^{-v} \nabla \cdot\left(e^{v} \nabla a\right)+a\left(a e^{v}+w\right) v-a z+\mu a\left(1-a e^{v}\right)\right\} \\
= & -\int_{\Omega} e^{v} \frac{|\nabla a|^{2}}{a^{2}}+\int_{\Omega} e^{v}(a-1)\left(a e^{v}+w\right) v-\int_{\Omega} e^{v}(a-1) z \\
& +\mu \int_{\Omega}\left(a e^{v}-e^{v}\right)\left(1-a e^{v}\right) \\
\leq & \int_{\Omega} e^{v} a\left(a e^{v}+w\right) v+\int_{\Omega} e^{v} z+\mu \int_{\Omega}\left(1-e^{v}\right)\left(1-a e^{v}\right) \\
& -\mu \int_{\Omega}\left(a e^{v}-1\right)^{2} \quad \text { for all } t>0
\end{aligned}
$$

due to the nonnegativity of $a, v, w$ and $z$. Here by the elementary inequality: $1-e^{-s} \leq s$ for all $s \geq 0$, we have $\left(e^{v}-1\right)=e^{v}\left(1-e^{-v}\right) \leq e^{v} v$, and thus in light of this and (4.9) we see that

$$
\begin{aligned}
\int_{\Omega} e^{v} a\left(a e^{v}+w\right) v+\int_{\Omega} e^{v} z & +\mu \int_{\Omega}\left(1-e^{v}\right)\left(1-a e^{v}\right) \\
& \leq \int_{\Omega} e^{v} a\left(a e^{v}+w\right) v+\int_{\Omega} e^{v} z+\mu \int_{\Omega} e^{v}\left(a e^{v}-1\right) v \\
& \leq \int_{\Omega}\left(a^{2} e^{2 v}+a w e^{v}+\mu a e^{2 v}\right) v+\int_{\Omega} e^{v} z \\
& \leq\left(c_{1}^{2} e^{2 c_{2}}+c_{1} c_{3} e^{c_{2}}+\mu c_{1} e^{2 c_{2}}\right) \int_{\Omega} v+e^{c_{2}} \int_{\Omega} z \quad \text { for all } t>0 .
\end{aligned}
$$

Therefore, we arrive at

$$
\frac{d}{d t} \int_{\Omega} e^{v}(a-1-\ln a)+\mu \int_{\Omega}\left(a e^{v}-1\right)^{2} \leq c_{6} \int_{\Omega} v+c_{7} \int_{\Omega} z \quad \text { for all } t>0
$$

with $c_{6}:=c_{1}^{2} e^{2 c_{2}}+c_{1} c_{3} e^{c_{2}}+\mu c_{1} e^{2 c_{2}}$ and $c_{7}:=e^{c_{2}}$. Integrating this in time entails that

$$
\begin{aligned}
\int_{\Omega} e^{v(\cdot, t)}\{a(\cdot, t)-1-\ln a(\cdot, t)\} & +\mu \int_{0}^{t} \int_{\Omega}\left(a e^{v}-1\right)^{2} \\
& \leq \int_{\Omega} e^{v_{0}}\left(a_{0}-1-\ln a_{0}\right)+c_{6} \int_{0}^{t} \int_{\Omega} v+c_{7} \int_{0}^{t} \int_{\Omega} z \\
& \leq \int_{\Omega} e^{v_{0}}\left(a_{0}-1-\ln a_{0}\right)+c_{6} \int_{0}^{\infty} \int_{\Omega} v+c_{7} \int_{0}^{\infty} \int_{\Omega} z \\
& =\int_{\Omega} e^{v_{0}}\left(a_{0}-1-\ln a_{0}\right)+c_{4} c_{6}+c_{5} c_{7} \quad \text { for all } t>0 .
\end{aligned}
$$


Since $s-1-\ln s \geq 0$ for all $s>0$ as mentioned before and since $a(x, t)>0$ in $\Omega \times(0, \infty)$ by Lemma 2.1, this implies that

$$
\int_{0}^{\infty} \int_{\Omega}\left(a e^{v}-1\right)^{2} \leq c_{8}:=\frac{1}{\mu} \cdot\left\{\int_{\Omega} e^{v_{0}}\left(a_{0}-1-\ln a_{0}\right)+c_{4} c_{6}+c_{5} c_{7}\right\}<\infty
$$

thanks to $\mu>0$ and the fact that $a_{0}:=u_{0} e^{-v_{0}}>0$ by (1.2), and thereby completes the proof.

In order to improve the above weak stabilization information, we need further regularity properties of $a$, which can be obtained through another testing procedure.

Lemma 4.5 Let $\beta<1$, then

$$
\int_{0}^{\infty} \int_{\Omega} a_{t}^{2}<\infty
$$

and

$$
\sup _{t>0} \int_{\Omega}|\nabla a(\cdot, t)|^{2}<\infty
$$

Proof. Lemma 3.4, Lemma 4.1 and Corollary 4.3 warrant the boundedness of $a, v, w$ and $z$ as well as the exponential decay of $v$ and $z$, which enables us to find $c_{i}>0, i \in\{1, \cdots, 5\}$, fulfilling

$$
2 e^{v} a^{2}\left(a e^{v}+w^{2}\right) \leq c_{1}, \quad 2 e^{v} a^{2} \leq c_{2} \quad \text { and } \quad e^{v} a^{2} \leq c_{3} \quad \text { in } \Omega \times(0, \infty)
$$

and moreover

$$
\int_{0}^{\infty} \int_{\Omega} v^{2} \leq c_{4} \quad \text { and } \quad \int_{0}^{\infty} \int_{\Omega} z^{2} \leq c_{5}
$$

Lemma 4.4 also provides $c_{6}>0$ satisfying

$$
\int_{0}^{\infty} \int_{\Omega}\left(a e^{v}-1\right)^{2}<c_{5}
$$

Next, multiplying the first equation in (3.2) by $e^{v} a_{t}$ and integrating by parts, we have

$$
\begin{aligned}
\int_{\Omega} e^{v} a_{t}^{2} & =\int_{\Omega} a_{t} \nabla \cdot\left(e^{v} \nabla a\right)+\int_{\Omega} e^{v} a_{t} a\left(a e^{v}+w\right) v-\int_{\Omega} e^{v} a_{t} a z+\mu \int_{\Omega} e^{v} a_{t} a\left(1-a e^{v}\right) \\
& =-\int_{\Omega} e^{v} \nabla a \cdot \nabla a_{t}+\int_{\Omega} e^{v} a_{t} a\left(a e^{v}+w\right) v-\int_{\Omega} e^{v} a_{t} a z+\mu \int_{\Omega} e^{v} a_{t} a\left(1-a e^{v}\right)
\end{aligned}
$$

for all $t>0$, where by nonpositivity of $v_{t}$,

$$
\begin{aligned}
-\int_{\Omega} e^{v} \nabla a \cdot \nabla a_{t} & =-\frac{1}{2} \int_{\Omega} e^{v} \partial_{t}|\nabla a|^{2} \\
& =-\frac{1}{2} \frac{d}{d t} \int_{\Omega} e^{v}|\nabla a|^{2}+\frac{1}{2} \int_{\Omega} e^{v}|\nabla a|^{2} v_{t} \\
& \leq-\frac{1}{2} \frac{d}{d t} \int_{\Omega} e^{v}|\nabla a|^{2} \quad \text { for all } t>0 .
\end{aligned}
$$


Moreover, in view of Young's inequality and (4.13),

$$
\begin{aligned}
\int_{\Omega} e^{v} a_{t} a\left(a e^{v}+w\right) v & \leq \frac{1}{8} \int_{\Omega} e^{v} a_{t}^{2}+2 \int_{\Omega} e^{v} a^{2}\left(a e^{v}+w\right)^{2} v^{2} \\
& \leq \frac{1}{8} \int_{\Omega} e^{v} a_{t}^{2}+c_{1} \int_{\Omega} v^{2} \quad \text { for all } t>0
\end{aligned}
$$

and similarly,

$$
\begin{aligned}
-\int_{\Omega} e^{v} a_{t} a z & \leq \frac{1}{8} \int_{\Omega} e^{v} a_{t}^{2}+2 \int_{\Omega} e^{v} a^{2} z^{2} \\
& \leq \frac{1}{8} \int_{\Omega} e^{v} a_{t}^{2}+c_{2} \int_{\Omega} z^{2} \quad \text { for all } t>0
\end{aligned}
$$

as well as

$$
\begin{aligned}
\mu \int_{\Omega} e^{v} a_{t} a\left(1-a e^{v}\right) & \leq \frac{1}{4} \int_{\Omega} e^{v} a_{t}^{2}+\mu^{2} \int_{\Omega} e^{v} a^{2}\left(1-a e^{v}\right)^{2} \\
& \leq \frac{1}{4} \int_{\Omega} e^{v} a_{t}^{2}+\mu^{2} c_{3} \int_{\Omega}\left(1-a e^{v}\right)^{2} \quad \text { for all } t>0 .
\end{aligned}
$$

Collecting (4.16)-(4.20) and integrating in time we obtain

$$
\begin{aligned}
\frac{1}{2} \int_{0}^{t} \int_{\Omega} e^{v} a_{t}^{2} & +\frac{1}{2} \int_{\Omega} e^{v(\cdot, t)}|\nabla a(\cdot, t)|^{2} \\
& \leq \frac{1}{2} \int_{\Omega} e^{v_{0}}\left|\nabla a_{0}\right|^{2}+c_{1} \int_{0}^{t} \int_{\Omega} v^{2}+c_{2} \int_{0}^{t} \int_{\Omega} z^{2}+\mu^{2} c_{3} \int_{0}^{t} \int_{\Omega}\left(1-a e^{v}\right)^{2} \\
& \leq \frac{1}{2} \int_{\Omega} e^{v_{0}}\left|\nabla a_{0}\right|^{2}+c_{1} \int_{0}^{\infty} \int_{\Omega} v^{2}+c_{2} \int_{0}^{\infty} \int_{\Omega} z^{2}+\mu^{2} c_{3} \int_{0}^{\infty} \int_{\Omega}\left(1-a e^{v}\right)^{2} \\
& \leq \frac{1}{2} \int_{\Omega} e^{v_{0}}\left|\nabla a_{0}\right|^{2}+c_{1} c_{4}+c_{2} c_{5}+\mu^{2} c_{3} c_{6} \quad \text { for all } t>0
\end{aligned}
$$

due to (4.14) and (4.15), which immediately entails (4.11) and (4.12) because $e^{v} \geq 1$.

Now using Lemma 4.4 in conjunction with the compactness properties implied by Lemma 4.5 we can claim the following $L^{2}$ stabilization feature of $a$.

Lemma 4.6 If $\beta<1$, then we have

$$
\|a(\cdot, t)-1\|_{L^{2}(\Omega)} \rightarrow 0 \quad \text { as } t \rightarrow \infty .
$$

Proof. $\quad$ Combining Lemma 4.4 with Lemma 3.4 and Corollary 4.3 we readily see that

$$
\int_{0}^{\infty} \int_{\Omega}(a-1)^{2}<\infty
$$

Relying on this basic decay information together with the relative compactness properties implied by (4.11) and (4.12), we use a straightforward argument by contradiction to achieve (4.21); we may refer to [15, Lemma 3.5] for details in a quite similar setting.

A simple interpolation yields a slightly stronger convergence statement in the sense: 
Lemma 4.7 Let $\beta<1$. Then the solution of (1.1) has the property

$$
u(\cdot, t) \rightarrow 1 \quad \text { in } L^{p}(\Omega) \quad \text { for all } p \in(2, \infty) \quad \text { as } t \rightarrow \infty .
$$

Proof. Since

$$
\begin{aligned}
\|u-1\|_{L^{p}(\Omega)} & =\|u-1\|_{L^{\infty}(\Omega)}^{\frac{p-2}{p}}\|u-1\|_{L^{2}(\Omega)}^{\frac{2}{p}} \\
& =\left\|e^{v} a-1\right\|_{L^{\infty}(\Omega)}^{\frac{p-2}{p}}\left\|e^{v} a-1\right\|_{L^{2}(\Omega)}^{\frac{2}{p}} \\
& \leq\left\{e^{\left.\left\|v_{0}\right\|_{L^{\infty}(\Omega)}\|a\|_{L^{\infty}(\Omega)}+1\right\}^{\frac{p-2}{p}}} \cdot\left\{\|a-1\|_{L^{2}(\Omega)}+\left\|e^{v} a\left(1-e^{-v}\right)\right\|_{L^{2}(\Omega)}\right\}^{\frac{2}{p}}\right. \\
& \leq\left\{e^{\left\|v_{0}\right\|_{L^{\infty}(\Omega)}\|a\|_{L^{\infty}(\Omega)}+1}\right\}^{\frac{p-2}{p}} \cdot\left\{\|a-1\|_{L^{2}(\Omega)}+e^{\left.\left\|v_{0}\right\|_{L^{\infty}(\Omega)}\|a\|_{L^{\infty}(\Omega)}\|v\|_{L^{2}(\Omega)}\right\}^{\frac{2}{p}}}\right.
\end{aligned}
$$

for all $t>0$ due to the Hölder inequality, (3.1), (2.1) and the basic inequality $1-e^{-s} \leq s$ for all $s \geq 0$, this straightly results from Lemma 4.6. Corollary 4.3 and Lemma 3.4.

Up to now, we have achieved all goals in Theorem 1.1 .

Proof of Theorem 1.1. This immediately is the outcomes of Lemma 2.1, Lemma 3.4, Lemma 4.1, Corollary 4.3 and Lemma 4.7.

\section{References}

[1] Alzahrani, T., Raluca Eftimie, R., Dumitru Trucu, D.: Multiscale modelling of cancer response to oncolytic viral therapy. Math. Biosci. 310, 76-95 (2019)

[2] Coffey, M.C., Strong, J.E., Forsyth, P.A., Lee, P.W.K.: Reovirus therapy of tumors with activated Ras pathways. Science 282, 1332-1334 (1998)

[3] Fontelos, M.A., Friedman, A., Hu, B.: Mathematical analysis of a model for the initiation of angiogenesis. SIAM J. Math. Anal. 33, 1330-1355 (2002)

[4] Friedman, A., Tello, J.I.: Stability of solutions of chemotaxis equations in reinforced random walks. J. Math. Anal. Appl. 272, 138-163 (2002)

[5] Herrero, M.A., Velázquez, J.J.L.: A blow-up mechanism for a chemotaxis model. Ann. Scuola Normale Superiore Pisa 24, 633-683 (1997)

[6] Jain, R.: Barriers to drug delivery in solid tumors. Sci. Am. 271, 58-65 (1994)

[7] Lițcanu, G., Morales-Rodrigo, C.: Asymptotic behavior of global solutions to a model of cell invasion. Math. Models Methods Appl. Sci. 20, 1721-1758 (2010)

[8] Pang, P.Y.H., Wang, Y.: Global boundedness of solutions to a chemotaxis-haptotaxis model with tissue remodeling. Math. Mod. Meth. Appl. Sci. 28, 2211-2235 (2018) 
[9] Russell, S.J., Peng, K.-W., Bell, J. C.: Oncolytic virotherapy. Nature Biotechnology 30, 658-670 (2012)

[10] Swabb, E.A., Wei, J., Gullino, P.M.: Diffusion and convection in normal and neoplastic tissues. Cancer Res. 34, 2814-2822 (1974)

[11] TAO, Y., WinkleR, M.: Energy-type estimates and global solvability in a two-dimensional chemotaxis-haptotaxis model with remodeling of non-diffusible attractant. J. Differential Eq. 257, 784-815 (2014)

[12] TAO, Y., Winkler, M.: Large time behavior in a mutidimensional chemotaxis-haptotaxis model with slow signal diffusion. SIAM J. Math. Anal. 47, 4229-4250 (2015)

[13] TaO, Y., Winkler, M.: Blow-up prevention by quadratic degradation in a two-dimensional Keller-Segel-Navier-Stokes system. Z. Angew. Math. Phys. 67, Art. 138 (2016)

[14] TaO, Y., Winkler, M.: Global classical solutions to a doubly haptotactic cross-diffusion system modeling oncolytic virotherapy. J. Differential Equations 268, 4973-4997 (2020)

[15] TaO, Y., Winkler, M.: Critical mass for infinite-time blow-up in a haptotaxis system with nonlinear zero-order interaction. Discr. Cont. Dyn. Syst. A, to appear

[16] TAO, Y., Winkler, M.: A critical virus production rate for blow-up suppression in a haptotatxis model for oncolytic virotherapy. Nonlinear Analysis 198, Art. 111870 (2020)

[17] Walker, C., WebB, G.F.: Global existence of classical solutions for a haptotaxis model. SIAM J. Math. Anal. 38, 1694-1713 (2007)

[18] Winkler, M.: Aggregation vs. global diffusive behavior in the higher-dimensional Keller-Segel model. J. Differential Eq. 248, 2889-2905 (2010)

[19] Winkler, M.: Finite-time blow-up in the higher-dimensional parabolic-parabolic Keller-Segel system. J. Math. Pures Appl. 100, 748-767 (2013), arXiv:1112.4156v1

[20] Winkler, M.: Singular structure formation in a degenerate haptotaxis model involving myopic diffusion. J. Math. Pures Appl. 112, 118-169 (2018)

[21] Winkler, M., Surulescu, C.: A global weak solutions to a strongly degenerate haptotaxis model. Commun. Math. Sci. 15, 1581-1616 (2017)

[22] Zhigun, A., Surulescu, C., Uatay, A.: Global existence for a degenerate haptotaxis model of cancer invasion. Z. Angew. Math. Phys. 67, Art. 146, 29 pp (2016) 